\title{
Numerical Simulation of Water Waves' Modulational Instability under the Effects of Wind's Stress and Gravity Force Relaxation
}

\author{
Théodule Nkoa Nkomom¹, César Mbane Biouele ${ }^{1}$, Jeannot Mane Mane ${ }^{2}$ \\ ${ }^{1}$ Laboratory of Earth's Atmosphere Physics, Department of Physics, University of Yaoundé I, Yaoundé, \\ Cameroun \\ ${ }^{2}$ Ecole Nationale Supérieure Polytechnique (National Advanced School of Engineering), Department of \\ Mathematics and Physical Sciences, University of Yaoundé I, Yaoundé, Cameroon \\ Email: *cesar.mbane@yahoo.fr
}

Received 28 December 2015; accepted 26 January 2016; published 29 January 2016

Copyright (C) 2016 by authors and Scientific Research Publishing Inc.

This work is licensed under the Creative Commons Attribution International License (CC BY).

http://creativecommons.org/licenses/by/4.0/

c) (i) Open Access

\begin{abstract}
The waves driven by the wind do not move on the water as ordinarily done by sailboats. Indeed, the movement of the waves driven by the wind is more complex than the sailboats' translation movement that we know. The movement of the wave in our particular case results from the chainjob done by wind's stress and gravity forces: material is collected upstream (erosion phenomenon) and then deposited on the wave's summit by the wind. This material deposited on the summit of the wave by the wind is then removed and dispatched on the downstream side of the wave by gravity forces. As always happens in any chain-job: if the wind works faster than gravity forces, great accumulation of material will occur at the summit of the wave that will lead to an increase in its (the wave in this case) height. If conversely the wind works more slowly, a deficit in material delivery will occur and gravity force goes directly to remove material on the wave's summit and lead to a decrease in its height. In terms of Mechanics, we know that the main obstacle that can seriously disturb the work of the wind is the unavailability of water or so its viscosity. Given the complexity of the process to be studied, it seemed necessary for us to make a use of modulational instability theories such as the standard NLSE in order to better understand the contribution of wind and water viscosity to modulations of driven waves' amplitudes (or phases): modulations which sometimes can accidentally trigger unpredictable rogue waves.
\end{abstract}

\section{Keywords}

Waves Driven by the Wind, Standard Nonlinear Schrödinger Equation, Modulations of Driven Waves' Amplitudes or Phases, Unpredictable Rogue Waves

\footnotetext{
"Corresponding author.
}

How to cite this paper: Nkoa Nkomom, T., Mbane Biouele, C. and Mane Mane, J. (2016) Numerical Simulation of Water Waves' Modulational Instability under the Effects of Wind's Stress and Gravity Force Relaxation. Open Journal of Marine Science, 6, 93-102. http://dx.doi.org/10.4236/ojms.2016.61009 


\section{Introduction}

Descriptions of unusually high waves appearing on the sea surface for a short time (freak, rogue or killer waves) have been considered as a part of marine folklore for a long time. A number of instrumental registrations have appeared recently making the community to pay more attention to this problem and to reconsider known observations of freak waves whose existences are now universally recognized [1]-[21]. Furthermore, images on the extent of damage caused by these monsters of the ocean are available. However, the physics of processes responsible for the formation (or origin) and propagation of these phenomena as well as their prediction is not completely understood. Contrary to developed country researchers' opinion, physics of spectacular phenomenon like rogue waves is not easily obtainable by the only use of advance data provided by high technology equipment like: Powerful Computers; sophisticated WIS (Weather Information Systems); Wind's Profilers; Radar Drones; Lidar Drones; Satellites; etc. Indeed, rogue waves are a combination of multi-spectral processes that occur under the thermodynamic and dynamic accuracy conditions. Mathematical models [22]-[25] offer more tremendous opportunities for understanding of the systems whose physics is, at the present level of our knowledge, difficult to obtain. To allow a better understanding of the behavior of rogue waves triggered by winds in terms of their origin and spatiotemporal evolution, that is, their motion and also in terms of mechanical transformations that these systems may suffer in their dealings with other systems, the standard nonlinear Schrödinger equation of off balance systems is implemented in the specific case of waves driven by wind and gravity forces. A mathematical model based on Navier-Stokes and Euler Lagrange equations coupled with assumptions derived from the literature on the nature of the training locations (or birth places) of rogue waves is developed to enhance the wind and gravity force effects on the behavior of surface waves. Given the complexity of the process to be studied, it seemed necessary for us to make a use of modulational instability theories such as the standard NLSE in order to better understand the contribution of wind and water viscosity to modulations of driven waves' amplitudes (or phases): modulations which sometimes can accidentally trigger unpredictable rogue waves.

\section{Navier-Stokes and Euler Lagrange Equations}

The general fluid continuity equation is given by:

$$
\frac{\partial \rho}{\partial t}+\frac{\partial \rho u}{\partial x}+\frac{\partial \rho v}{\partial y}+\frac{\partial \rho w}{\partial z}=0
$$

This leads to the continuity equation for an incompressible fluid

$$
\frac{\partial u}{\partial x}+\frac{\partial v}{\partial y}+\frac{\partial w}{\partial z}=0
$$

The kinematic boundary conditions

$$
\boldsymbol{V n}=0, \text { at } z=-H \text { and } z=\eta(x, y, t)
$$

where $\boldsymbol{n}$ is the unit vector normal to the boundary surfaces and $\eta(x, y, t)$ is the sea surface elevation. Hence:

$$
\left(u, v, w-\frac{\partial \eta}{\partial t}\right)\left(-\frac{\partial \eta}{\partial x}, \frac{\partial \eta}{\partial y}, 1\right)=0, \quad z=\eta \Rightarrow w=\frac{\partial \eta}{\partial t}+u \frac{\partial \eta}{\partial x}+v \frac{\partial \eta}{\partial y}
$$

Equation of motion in natural coordinates

$$
\frac{\partial \boldsymbol{V}}{\partial t}+(\boldsymbol{V} \nabla) \boldsymbol{V}=\boldsymbol{v}((\nabla \nabla) \boldsymbol{V})-\frac{1}{\rho} \nabla p+\boldsymbol{g}
$$

According to (5): solutions of the equation of motion depends mainly on pressure $P$ (closely related to the prevailing winder over the ocean); gravity g and water viscosity $(v)$. Only mathematical models (theories) that incorporate best these different parameters will next be considered. 


\section{Weakly Nonlinear Approach: The Nonlinear Schrödinger Equation}

Under the effects of wind and gravity, the wave moves through successive deformations of its surface (Figure 1). This situation is perfectly described by the Nonlinear Schrödinger Equation (NLSE) which can be obtained from the fully nonlinear potential theory by using the multi-scale method [1]-[7]. The Taylor developing-series allows the NLSE to be expanded around a small wave steepness $\boldsymbol{\varepsilon}$. Thereafter, the equation obtained is a third order in $\varepsilon$ approximation of the system formed by equations 1 to 4 . To investigate both damping and amplification effects on the Benjamin-Feir instability, we have use the forced and damped nonlinear Schrödinger equation ( $f d N L S E)$,

$$
i\left(\psi_{t}+c_{g} \psi_{x y}-\frac{\omega_{0}}{8 k_{0}^{2}} \psi_{(x x, y y)}-2 \omega_{0} k_{0}^{2}|\psi|^{2} \psi\right)=i \frac{W \omega_{0} k_{0}}{2 g \rho} \psi-2 i v k_{0}^{2} \psi
$$

Herein, $W=\rho_{\text {air }} \beta u_{*}^{2} / k^{2}$ represents the wind effect, $c_{g}=\omega_{0} / 2 k_{0}$ is the group velocity of the carrier wave and $v, \rho, \rho_{\text {air }}, g, u_{*}$, and $k$ are well-known parameters [1]-[12]. Equation (6) describes the spatial and temporal evolution of the envelope of the surface elevation $\psi$, for weakly nonlinear and dispersive gravity waves on deep water when dissipation (due to viscosity), and amplification (due to wind), are considered. If considering the right hand side of this equation, it can be rewritten as

$$
i\left(\frac{W \omega_{0} k_{0}}{2 g \rho}-2 v k_{0}^{2}\right) \psi=i K \psi
$$

The stability of the envelope depends on the sign of $K$. For values of $K<0$, solutions are found to be stable, while for values of $K \geq 0$, solutions are unstable. Physically, they interpreted this result in terms of frequency of the carrier wave $\omega_{0}$ and friction velocity $u_{*}$ of the wind over the waves. We plotted (Figure 2 \& Figure 3 ) the critical curve separating stable envelopes from unstable envelopes. Namely, they showed that for a given friction velocity $u_{*}$, only carrier wave of frequency $\omega_{0}$ which satisfies the following condition are unstable to modulational perturbations

$$
i \frac{\partial \psi}{\partial T}-\frac{1}{8} \frac{\partial^{2} \psi}{\partial x^{2}}+\frac{1}{4} \frac{\partial^{2} \psi}{\partial y^{2}}-\frac{1}{2}\left|\psi^{2}\right| \psi=i K \psi
$$

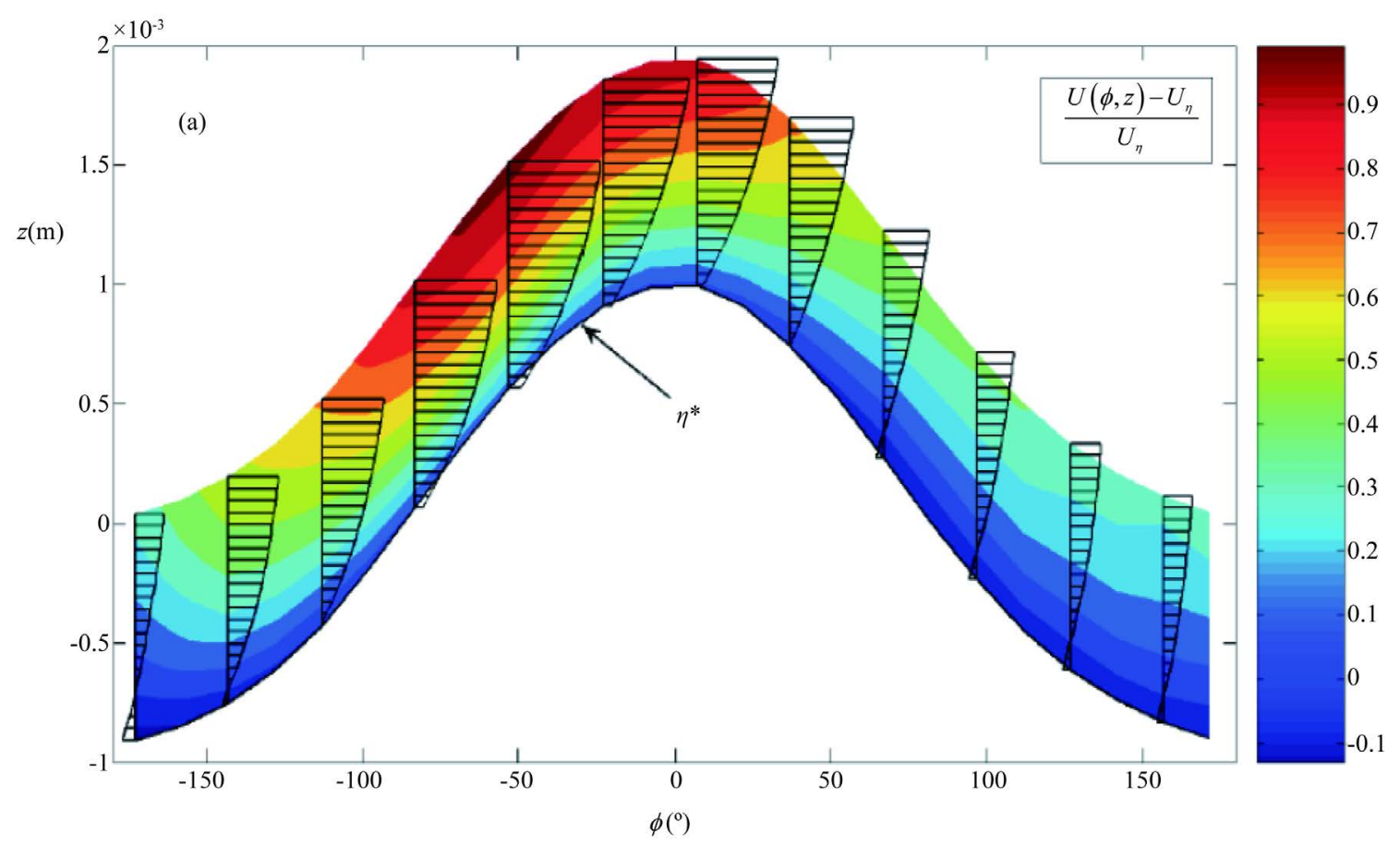

Figure 1. Driven wave’s velocity profile [1]. 


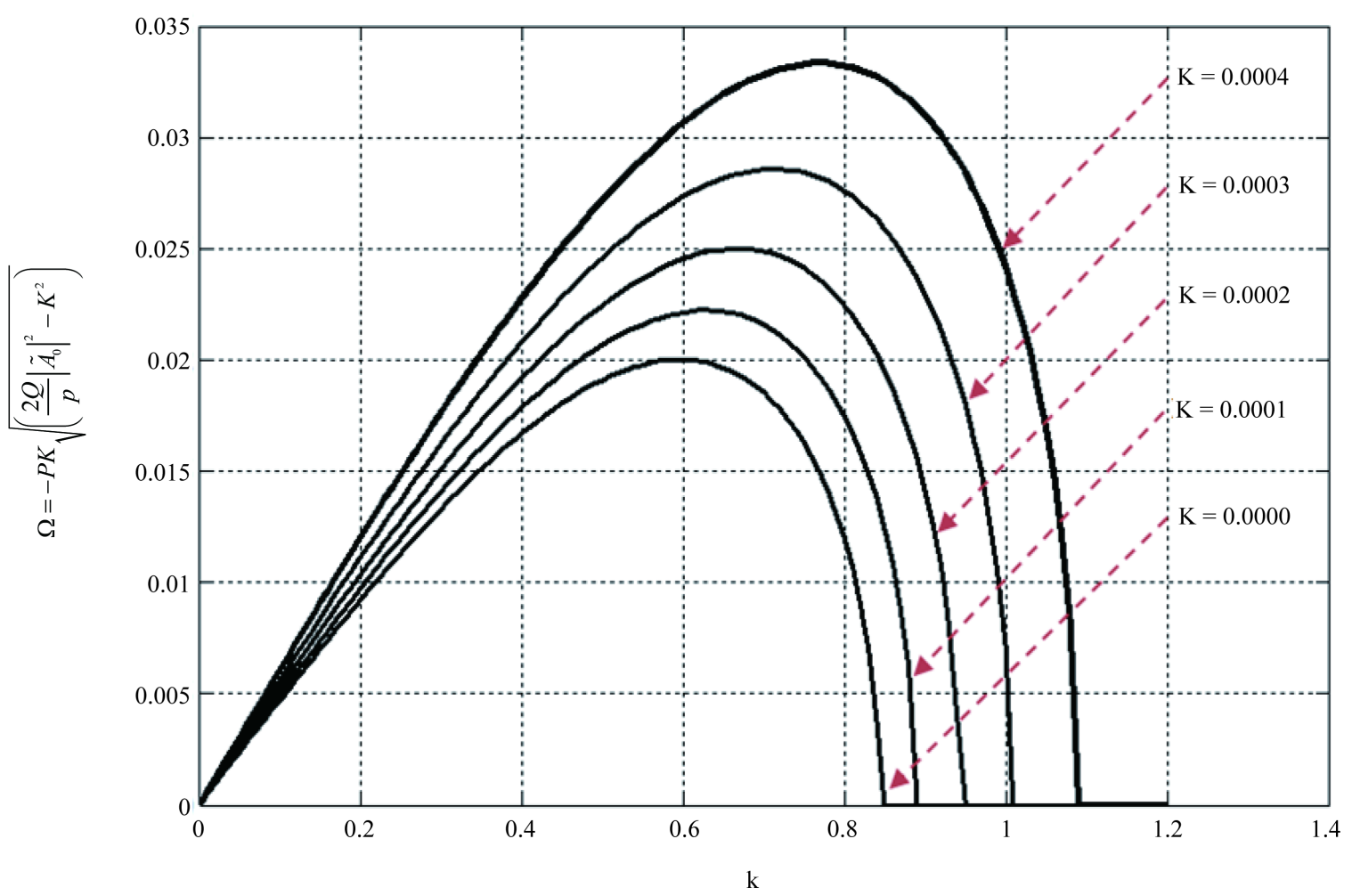

Figure 2. Growth rate versus of the wave number of the perturbation $\mathrm{k}$ for positive values of $K\left(A_{0}=0.2\right.$ and $\left.\theta=35\right)$.

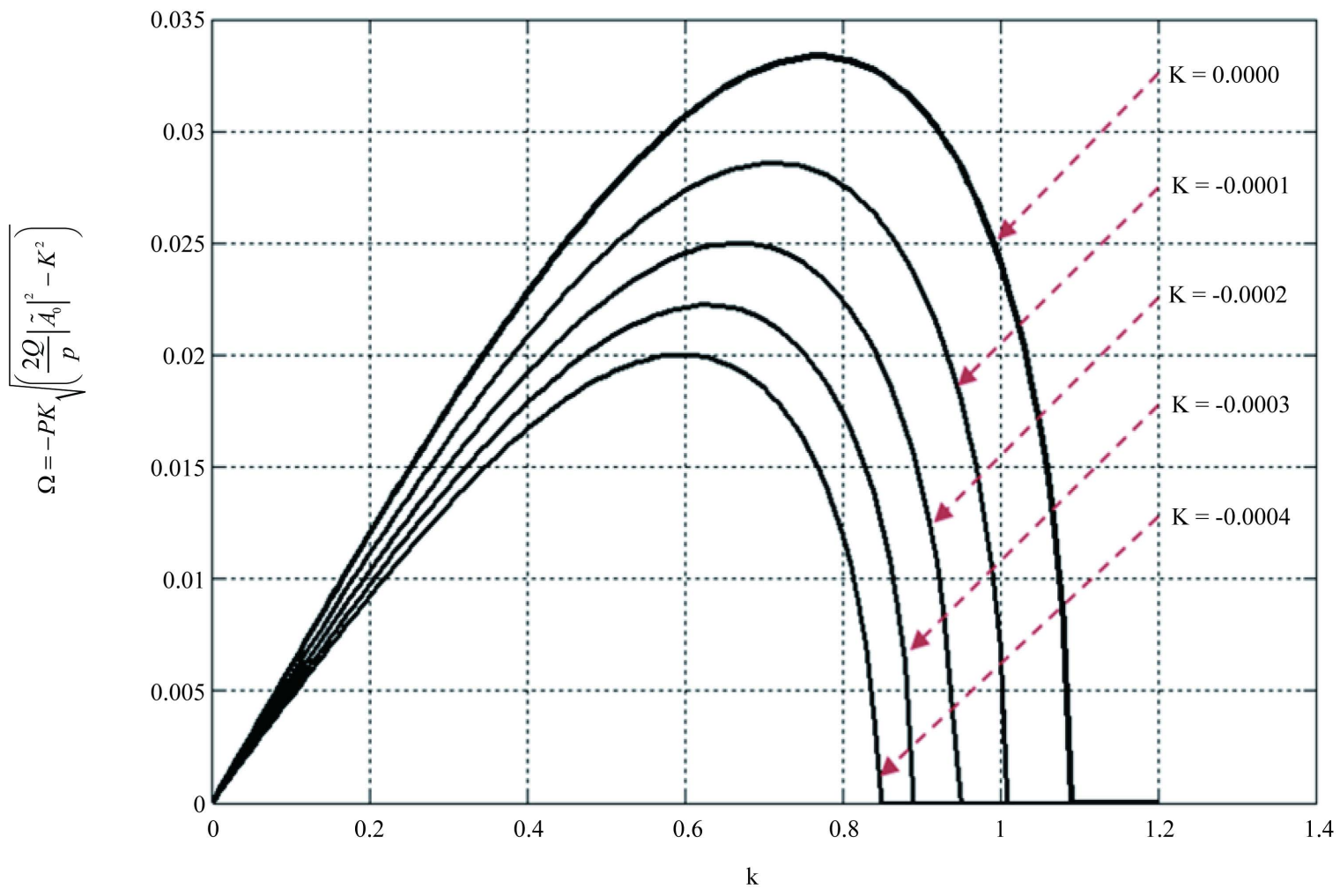

Figure 3. Growth rate versus of the wave number of the perturbation $\mathrm{k}$ for negative values of $K\left(A_{0}=0.2\right.$ and $\left.\theta=35\right)$. 
We can transform this Equation (8) to have a standard nonlinear Schrödinger equation in 3D.

$$
\eta(x, y, T)=\psi(x, y, t) \mathrm{e}^{-K T}
$$

And then introduce an oblique coordinates (S) associated with the direction of the spatial modulation

$$
S=x \cos \theta+y \sin \theta
$$

Substuting Equation (10) and (9) in (8) we obtain:

$$
i \frac{\partial \eta}{\partial t}+\frac{1}{8}\left(2-3 \cos ^{2} \theta\right) \frac{\partial^{2} \eta}{\partial S^{2}}-\frac{1}{2} \mathrm{e}^{2 K T}|\eta|^{2} \eta=0
$$

$K$ values are very small and this make possible to do Taylor expansion in first order of $\mathrm{e}^{2 K T}$ and obtain (12)

$$
i \frac{\partial \eta}{\partial t}+\frac{1}{8}\left(2-3 \cos ^{2} \theta\right) \frac{\partial^{2} \eta}{\partial S^{2}}-\frac{1}{2} \frac{1}{(1-2 K t)}|\eta|^{2} \eta=0
$$

Taking $Q^{\prime}=\frac{1}{2(1-2 K T)}$

Moving from the NLS equation to the standard NLS become possible if $q(T)=\frac{1}{2(1-2 K T)}$ is a linear function in T. We can therefore consider the following new variable:

$$
\xi=q(T) S \text { and } t=q(T) T
$$

And obtain

$$
A(\xi, t)=\frac{\eta(\xi, T)}{\sqrt{q(T)}} \exp \left[-\frac{K q(T) S^{2}}{2 P}\right]
$$

Therefore

$$
i \frac{\partial A}{\partial t}-P \frac{\partial^{2} A}{\partial \xi^{2}} Q|A|^{2} A=0
$$

where

$$
P=-\frac{1}{8}\left(2-3 \cos ^{2} \theta\right) \text { and } Q=\frac{1}{2}
$$

As said before, wave trains are unstable to small perturbation of other wave travelling in the same direction [2]. The standard stability analysis consist in linearizing around the monochromatic wave solution of the nonlinear Schrödinger equation

$$
\begin{aligned}
& A=\tilde{A} \mathrm{e}^{i Q|A|^{2}}+c C \\
& \tilde{A}=\tilde{A}_{0}+\varepsilon \tilde{A}_{1} \\
& \tilde{A}=\tilde{A}_{0} e^{i(K \xi-\Omega t)}+c C
\end{aligned}
$$

Here $K$ and $\Omega$ referring respectively to the perturbation wave number and frequency of the perturbation amplitude. Substituting this equation in Equation (8) we obtain the growth rate of instability:

$$
\Omega=-P K \sqrt{\left(\frac{2 Q^{\prime}}{P}\left|\tilde{A}_{0}\right|^{2}-K^{2}\right)}
$$

It is well known that one of the main effects of Modulational instability, which refers to the growth of certain modulation sideband of nonlinear plane waves propagating in a dispersive medium as deep water is a result of the interplay between nonlinearity, gravity and dispersion effects. In this case of forced/damped regime in deep water, instability is significantly influenced by $K$ (Figures 4-9). It is seen that the growth rate increases (or decreases) 


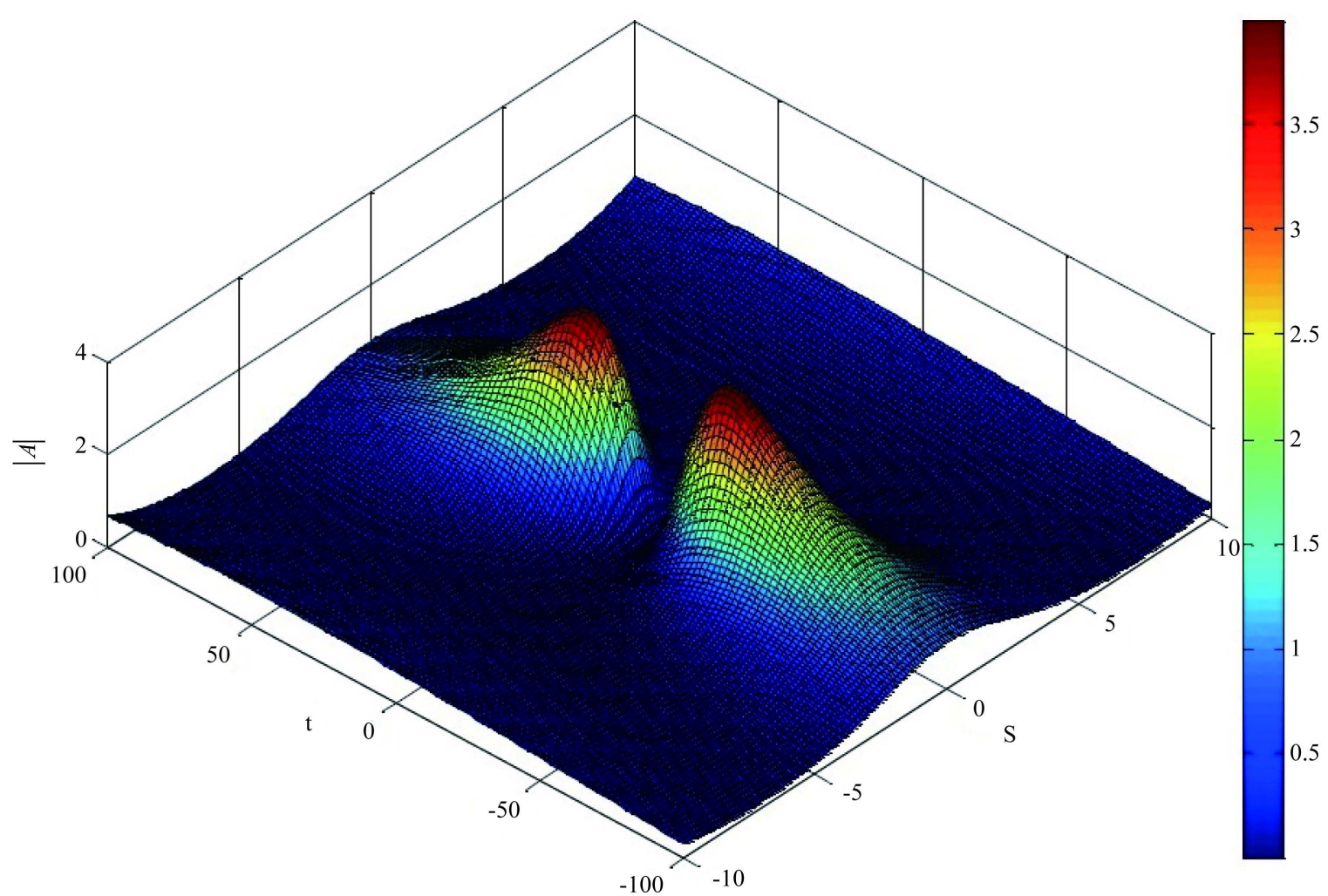

Figure 4. $\eta=\eta(S)$ at: $-100 \leq \mathrm{t} \leq 100$.

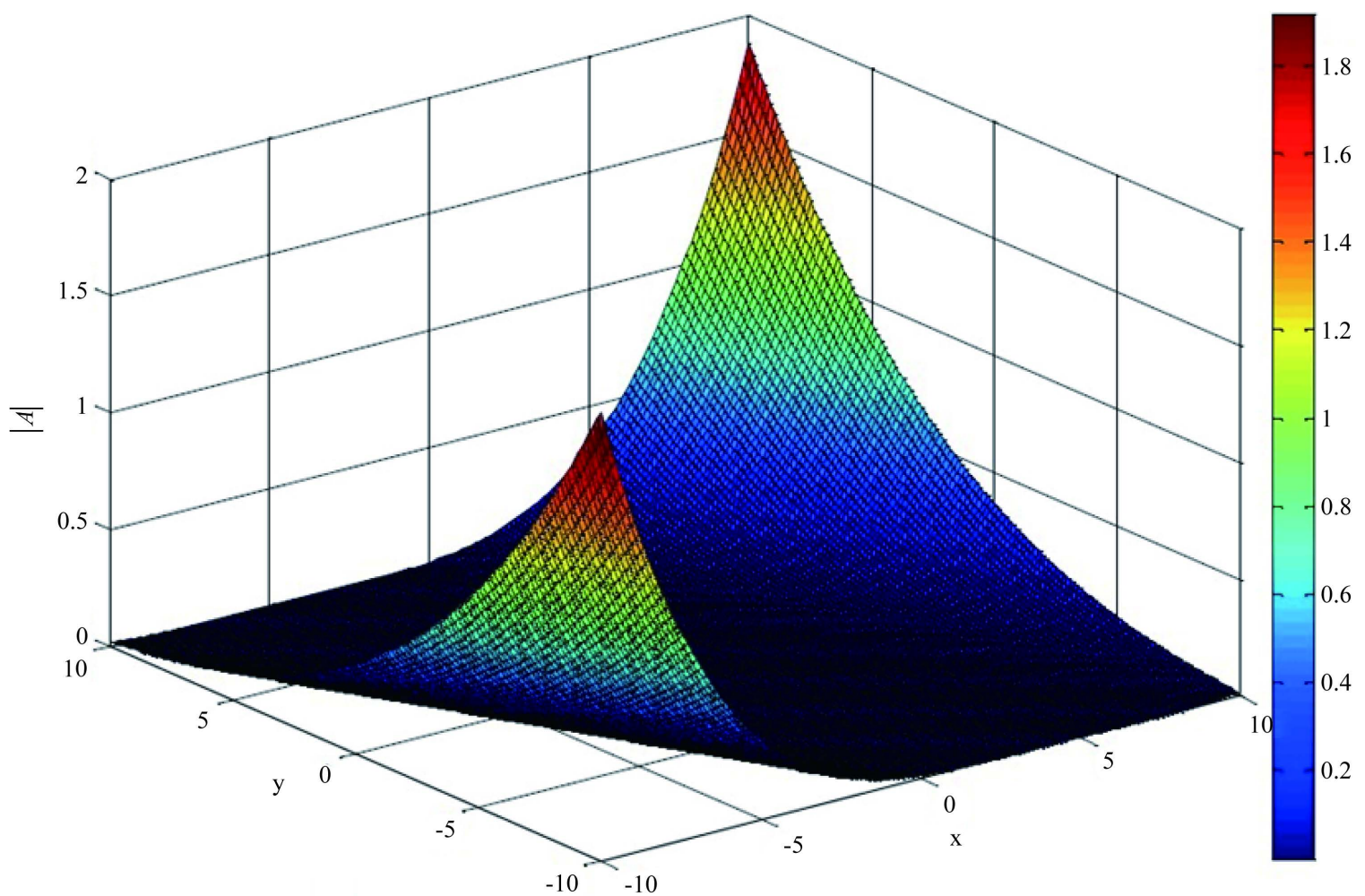

Figure 5. $\eta=\eta(x, y)$ at: $\mathrm{t}=0 ; \eta_{\max }=1.8$. 


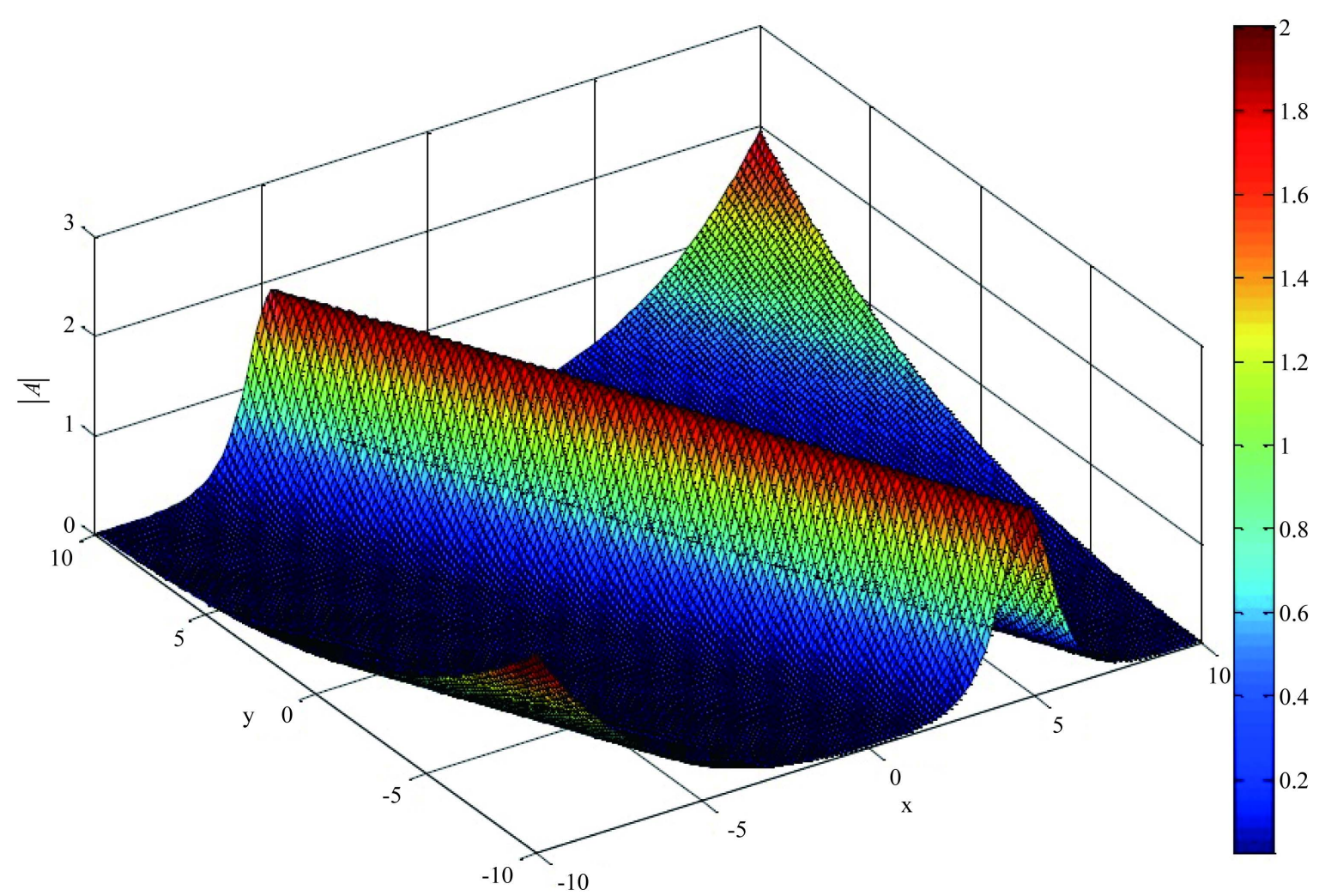

Figure 6. $\eta=\eta(x, y)$ at: $\mathrm{t}=10 ; \eta_{\max }=2.0$.

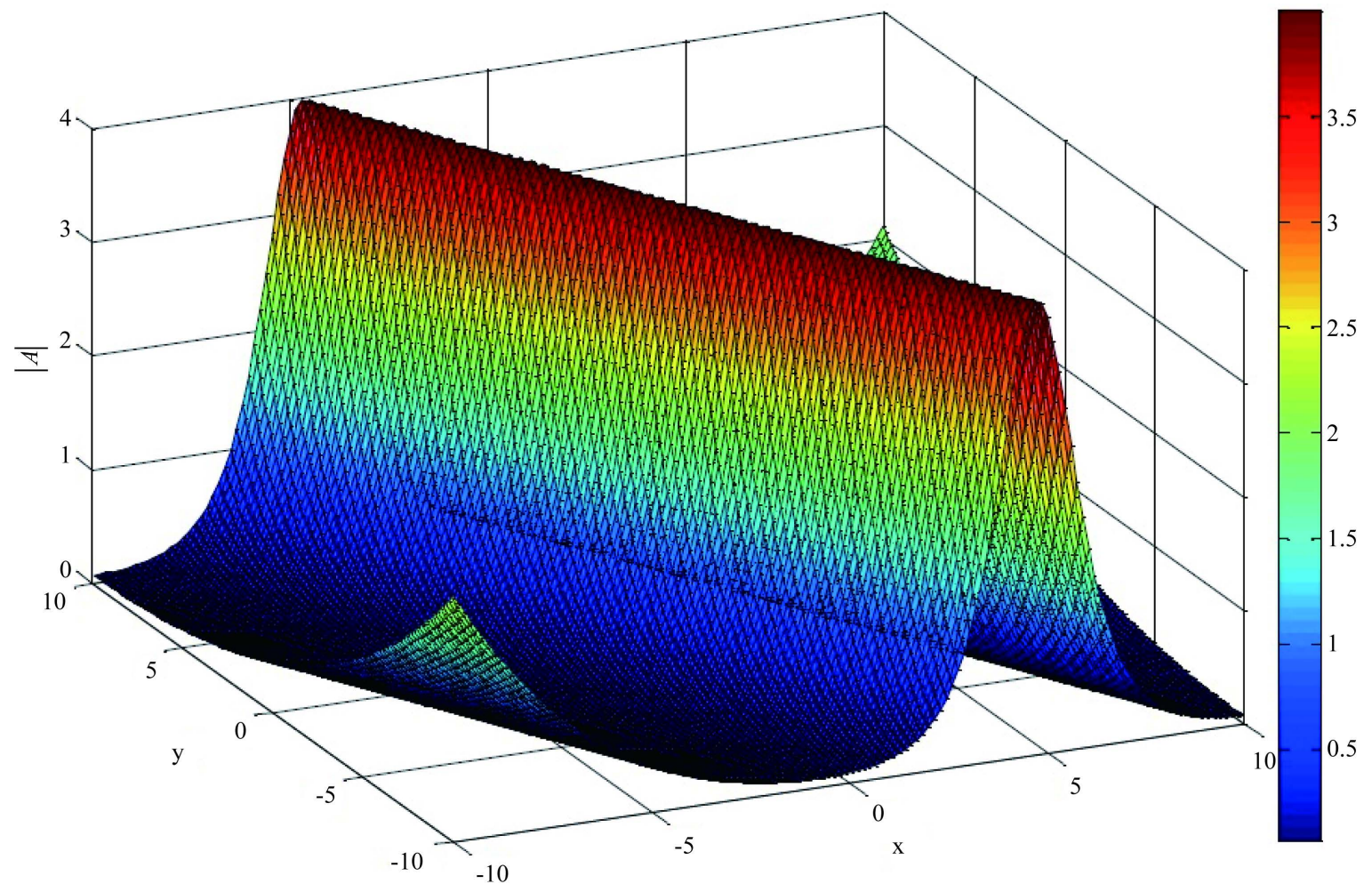

Figure 7. $\eta=\eta(x, y)$ at: $\mathrm{t}=25 ; \eta_{\max }=4.0$. 


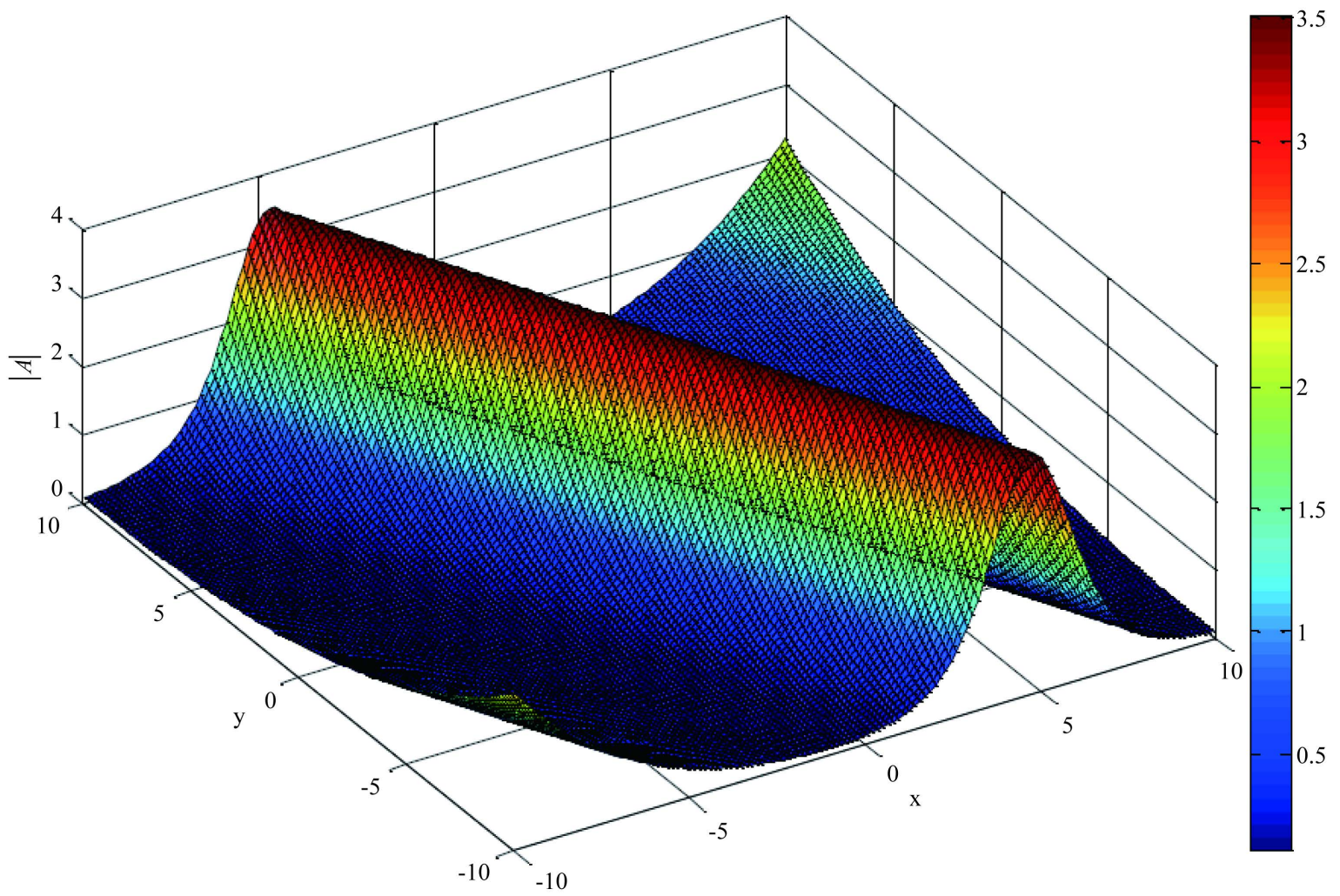

Figure 8. $\eta=\eta(x, y)$ at: $\mathrm{t}=35 ; \quad \eta_{\max } \cong 4$.

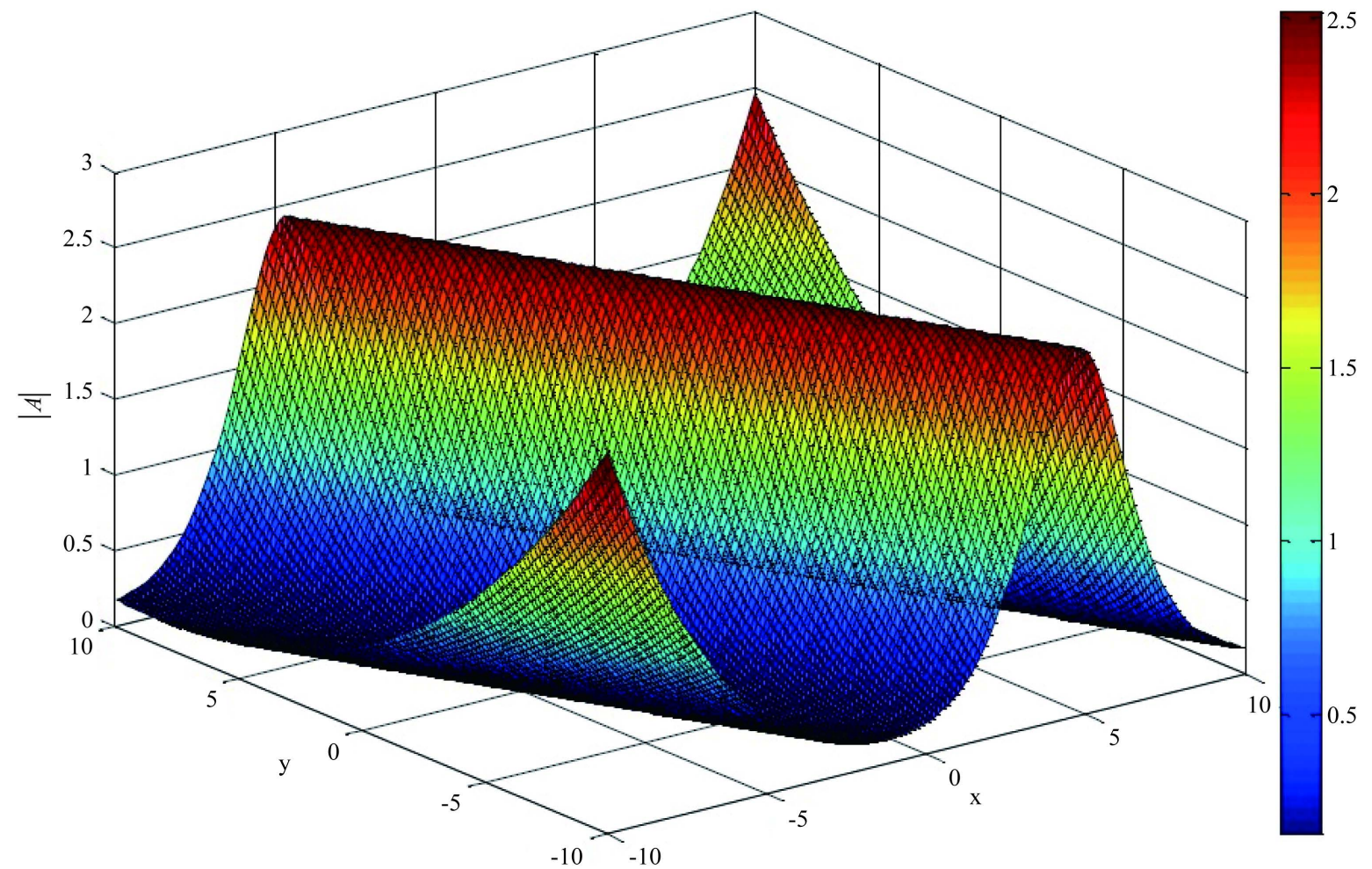

Figure 9. $\eta=\eta(x, y)$ at: $\mathrm{t}=50 ; \quad \eta_{\max 1} \cong 1.8, \eta_{\max 2}=2.4, \eta_{\max 3}=2.5$. 
with the increasing (decreasing) values of forcing/damping coefficient $K$. It is evident to say that wind effect destabilize the wave train in deep water in two dimensional directions however the dissipation stabilize the wave train.

\section{Conclusion}

The upsurges of abnormally large waves on the sea surface are due to complex physical mechanisms. In this paper, we discuss the modulational instability scenario of a single freak wave formation in 3D (i.e., time-space scales). One of the important properties of an unstable wave packet is the growth rate. Small amplitude modulation theory for the NLS equation predicts a number of interesting features about the nonlinear propagation of initially small amplitude sine wave modulations. One of the most important is shown in Figures 5-7 where carrier-wave's amplitude increases with respect to $\mathrm{t}$ (e.g., when comparing: $\eta(x, y, t=0)$ to $\eta(x, y, t=10)$ or $\eta(x, y, t=25)$. A later time after, the carrier wave's amplitude modulation escapes from our prediction (we observe the upsurge of amplitude's modulational instability. e.g., amplitude of the carrier wave at $t=35$ (Figure 8) becomes lesser than amplitude of the same carrier wave at $t=50$ (Figure 9) where $\mathbf{0 3}$ complete summits are observed). According to pertinent results obtained in this paper, wind alone cannot easily generate rogue waves; effect of winds combined with other physical mechanisms (e.g., viscosity and gravity force) may provide a fuller explanation of upsurge of freak wave phenomena. As wind blows over the ocean, its momentum is transferred to water molecules located at the surface of the sea. When strong winds (e.g., winds triggered by cyclones) blow in the opposing direction of the ocean current, its intensity might be strong enough to randomly generate rogue waves. Viscosity and gravity force contributions to the behavior of wind's carrier waves are well described by our adopted weakly nonlinear approach and the related NLSE (Nonlinear Schrödinger Equation).

\section{References}

[1] Touboul, J., Kharif, C., Pelinovsky, E. and Giovanangeli, J.P. (2008) On the Interaction of Wind and Steep Gravity Wave Groups Using Miles’ and Jeffreys’ Mechanisms. Nonlinear Processes in Geophysics, 15, 1023-1031. http://dx.doi.org/10.5194/npg-15-1023-2008

[2] Benjamin, T.B. (1967) Instability of Periodic Wave Trains in Nonlinear Dispersive Systems. Proceedings of the Royal Society of London, 299, 59-75. http://dx.doi.org/10.1098/rspa.1967.0123

[3] Karsten, T. and Igor, K. (2000) On Weakly Nonlinear Modulation of Waves on Deep Water. Physics of Fluids, 12, 24-32.

[4] Onorato, M., Osborne, A.R., Serio, M., Cavaleri, L. and Stanberg, T.C. (2004) Observation of Strongly Non-Gaussian Statistics for Random Sea Surface Gravity Waves in Wave Flume. Physical Review E, 70, Article ID: 067302. http://dx.doi.org/10.1103/PhysRevE.70.067302

[5] Socquet, H., Juglar, A., Dysthge, K., Trulsen, K., Krogstad, H.E. and Liu, J. (2005) Probability Distributions of Surface Waves during Spectral Change. Journal of Fluid Mechanics, 542, 1-21.

[6] Dyachenko, A.L. and Zakharov, V.E. (2005) Modulation Instability of Stokes Wave-Freak Wave. Journal of Experimental and Theoretical Physics, 81, 318-322. http://dx.doi.org/10.1134/1.1931010

[7] Kharif, C. and Pelinovsky, E. (2003) Physical Mechanism of the Rogue Wave Phenomenon. European Journal of Mechanics/B-Fluid, 22, 603-634.

[8] Wu, C.H. and Yao, A. (2004) Laboratory Measurements of Limiting Freak Waves on Current. Journal of Geophys-ical Research, 109, 1-18. http://dx.doi.org/10.1029/2004JC002612

[9] White, B.S. and Fomberg, B. (1998) On the Chance of Freak Waves at Sea. Journal of Fluid Mechanics, 355, $113-138$. http://dx.doi.org/10.1017/S0022112097007751

[10] Zakharov, V.E. and Kharitonov, N.G. (1970) Instability of Periodic Waves of Finite Amplitude on the Surface of a Deep Fluid. Journal of Applied Mechanics and Technical Physics, 11, 747-751. http://dx.doi.org/10.1007/BF00851899

[11] Shener, L. (2010) On Benjamin-Feir Instability and Evolution of Nonlinear Wave with Finite-Amplitude Side Bands. Natural Hazards and Earth System Sciences, 10, 2421-2427. http://dx.doi.org/10.5194/nhess-10-2421-2010

[12] Leblanc, S. (2008) Wind Forced Modulations of Finite Depth gravity Waves. Physics of Fluids, 20, Article ID: 116603. http://dx.doi.org/10.1063/1.3026551

[13] Batra, K., Sharma, R.P. and Verga, A.D. (2006) Stability Analysis on Nonlinear Evolution Patterns of Modulational Zakharov Equations. Journal of Plasma Physics, 72, 671-686. http://dx.doi.org/10.1017/S002237780500423X

[14] Banner, M.I. and Song, J.B. (2002) On Determining the Onset and Strength of Breaking for Deep Water Waves. Part ii: 
Influence of Wind Forcing and Surface Shear. Journal of Physical Oceanography, 32, 2559-2570. http://dx.doi.org/10.1175/1520-0485-32.9.2559

[15] Brown, M.G. and Jensen, A. (2001) Experiments on Focusing Unidirectional Water Waves. Journal of Geophysical Research, 106, 16917-16928. http://dx.doi.org/10.1029/2000JC000584

[16] Jeffreys, H. (1925) On the Formation of Wave by Wind. Proceedings of the Royal Society A, 107, 189-206. http://dx.doi.org/10.1098/rspa.1925.0015

[17] Philips, O.M. (1957) On the Interaction of Waves by Turbulent Wind. Journal of Fluid Mechanics, 2, 417-455. http://dx.doi.org/10.1017/S0022112057000233

[18] Makin, V.K., Branger, H., Peirson, W.L. and Giovanangeli, W.L. (2007) Stress above Wind-plus-Paddle Waves: Modeling of a Laboratory Experiment. Journal of Physical Oceanography, 37, 2824-2837. http://dx.doi.org/10.1175/2007JPO3550.1

[19] Shemer, L., Goulitski, K. and Kit, E. (2007) Evolution of Wide Spectrum Unidirectional Wave Groups in a Tank: An Experimental and Numerical Study. European Journal of Mechanics—B/Fluids, 26, 193-219. http://dx.doi.org/10.1016/j.euromechflu.2006.06.004

[20] Song, J.B. and Banner, M.I. (2002) On Determining the Onset and Strength of Breaking for Deep Water Waves, Part i: Unforced Irrotational Wave Groups. Journal of Physical Oceanography, 32, 2541-2558. http://dx.doi.org/10.1175/1520-0485-32.9.2541

[21] Stanton, T., Marshall, D. and Houghton, R. (1932) The Growth of Waves on Water Due to the Action of the Wind. Proceedings of the Royal Society A, 137, 283-293. http://dx.doi.org/10.1098/rspa.1932.0136

[22] Daika, A., Etoundi, H.M., Ngabireng, C.M. and Mbané-Biouélé, C. (2012) Application of Benjamin-Feir Equations to Tornadoes’ Rogue Waves Modulational Instability in Oceans. International Journal of Physical Sciences, 7, 60536061.

[23] Talon, M. (2006) Ondes de Surface. LIPTHE Paris VI-CNRS.

[24] Miles, J.W. (1996) Surface-Wave Generation: A Viscoelastic Model. Journal of Fluid Mechanics, 322, 131-145. http://dx.doi.org/10.1017/S002211209600273X

[25] Daika, A., Nkoa Nkomom, T. and Mbané Biouélé, C. (2014) Application of Stationnary Phase Method to Wind Stress and Breaking Impacts on Ocean Relatively High Waves. Open Journal of Marine Sciences, 4, 18-24. http://dx.doi.org/10.4236/ojms.2014.41003 